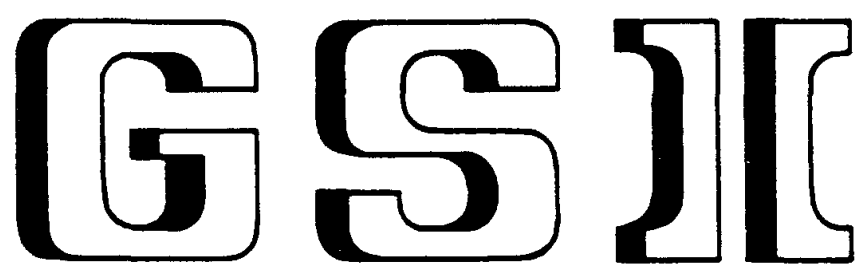

A NEW VME-BASED HIGH VOLTAGE POWER SUPPLY FOR LARGE PHOTOMULTIPLIER SYSTEMS

S. NEUMAIER, H.H. GUTBROD. T. HUBBELING, B.W. KOLB,

M.L. PURSCHKE. M. IPPOLITOV, V. MANKO, G. MGEBRISHVILI.

A. NIJANIN, Y. SIBIRYAK. A. TSVETKOV. A. VINOGRADOV.

C. BARLAG, C. BLUME. E.-M. BOHNE, D. BUCHER, A. CLAUSSEN,

T. PEITZMANN, G. SHEPERS, H. SCHLAGHECK

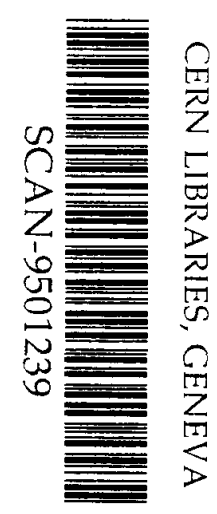

Gesellschaft für Schwerionenforschung $\mathrm{mbH}$ Postfach 110552 - D-64220 Darmstadt · Germany 


\section{A NEW VME-BASED HIGH VOLTAGE POWER SUPPLY FOR LARGE PHOTOMULTIPLIER SYSTEMS}

\section{S. NEUMAIER}

Division PPE, CERN, CH-1211 Genève 23

H.H. GUTBROD, T. HUBBELING, B.W. KOLB, M.L. PURSCHKE

GSI-Darmstadt, D-64220 Darmstadt

M. IPPOLITOV, V. MANKO, G. MGEBRISHVILI, A. NIJANIN, Y. SIBIRYAK, A. TSVETKOV, A. VINOGRADOV

Kurchatov Institute Moscow, Russia

C. BARLAG, C. BLUME, E.-M. BOHNE, D. BUCHER, A. ClAUSSEN, T. PEITZ-

MANN, G. SCHEPERS and H. SCHLAGHECK

University of Münster, D-48149 Münster

We describe a new high voltage power supply, developed for the leadglass calorimeter of the WA98 experiment at CERN. The high voltage is produced for each of the 10,080 photomultiplier tubes of the detector individually, by the same number of active bases with on-board Greinacher voltage multipliers. The full VME-based HV controller system, which addresses each base via bus cables once per second, is miniaturized and fits into a single VME crate. The main advantages of this approach are the low heat dissipation, the considerably reduced amount of cabling and cost, as well as the high stability and low noise of the system.

\section{Introduction}

New detector developments in high-energy physics as well as experiments searching for rare events often require photomultiplier (PM) systems of several thousands of channels. For such large PM systems the use of remote HV generators with $\mathrm{HV}$ cables and bases with resistive divider chains becomes inconvenient. The main disadvantages of conventional HV power supplies, e.g. the considerable amount of cabling and costs, the undesired heat dissipation as well as safety and control problems, led to new concepts for appropriate HV systems. Already in $1978 \mathrm{~L}$. Hubbeling suggested a new PM base with an on-board Greinacher voltage multiplier to reduce power consumption and cabling [1]. 
In recent years, similar developments were made by several groups $[2,3,4]$. Positive experiences with such approaches were reported from the ZEUS detector at HERA, which is equipped with about 12,000 photomultiplier tubes [4] and the D0 experiment at Fermilab [3].

The new HV system described in this paper has been developed for the leadglass calorimeter of the WA98 experiment, recently set up at the CERN SPS [5]. The experiment is dedicated to study ultrarelativistic heavy-ion collisions with the $160 \mathrm{~A} \cdot \mathrm{GeV}$ lead beam, which has become available at CERN in November 1994.

The segmented leadglass calorimeter is one of the major detectors of the WA98 experiment. It consists of 10,080 leadglass Čerenkov detectors of $4 \times 4 \times 40 \mathrm{~cm}^{3}$ each, with a photomultiplier tube read out for each module. The purpose of the spectrometer is the detection of photons and the precise reconstruction of neutral mesons, which requires an excellent energy resolution and hence, a better stability of the HV system than required for sampling calorimeters.

The main innovation of the new HV system which uses PM bases with on-board Greinacher voltage multipliers (also referred to as Cockcroft-Walton generators) is the capability to control up to $2048 \mathrm{HV}$ bases with a single slot VME controller card. In contrast to similar developments $[2,3,4]$, the use of bus cables and controllers which continuously cycle through all bases, allows a further significant reduction of cabling, as well as a minimum amount of space required for the control system. The controllers for the 10,080 channels of our experiment occupy less than half of a VME crate.

In addition, the new HV system allows a fast and easy way to set and control the high voltages and simplifies the identification and disabling of bad channels.

The leadglass calorimeter assembled with the new system was finished, successfully tested and calibrated with electron beams at the CERN SPS in spring 1994. A detailed description of the leadglass calorimeter of the WA98 experiment will be given elsewhere. The good stability of the new system as well as improvements in the design of the detector led to a slightly improved energy resolution compared to previous leadglass detector arrays with conventional HV systems.

\section{The new PM base}

The new photomultiplier base, designed by L. Hubbeling [6] is subdivided into two functional units: the high voltage card, consisting of the Greinacher voltage multiplier chain and the receiver card with bus receiver, oscillator, regulator, the feedback path, and the connection to a bus cable which connects groups of bases with the controller. The working principle of the PM base is illustrated in Fig. 1.

Each receiver card is equipped with an address decoder chip, that gives a valid transmission signal when its own 9-bit address (set by jumpers) is found twice in succession. The 9-bit address allows for a maximum of 512 bases of one group to be addressed and controlled by a remote control unit.

The valid transmission signal is stretched to about $1 \mathrm{~ms}$. During this period, an analog reference voltage (1/1000 of the demand cathode voltage) generated by the 


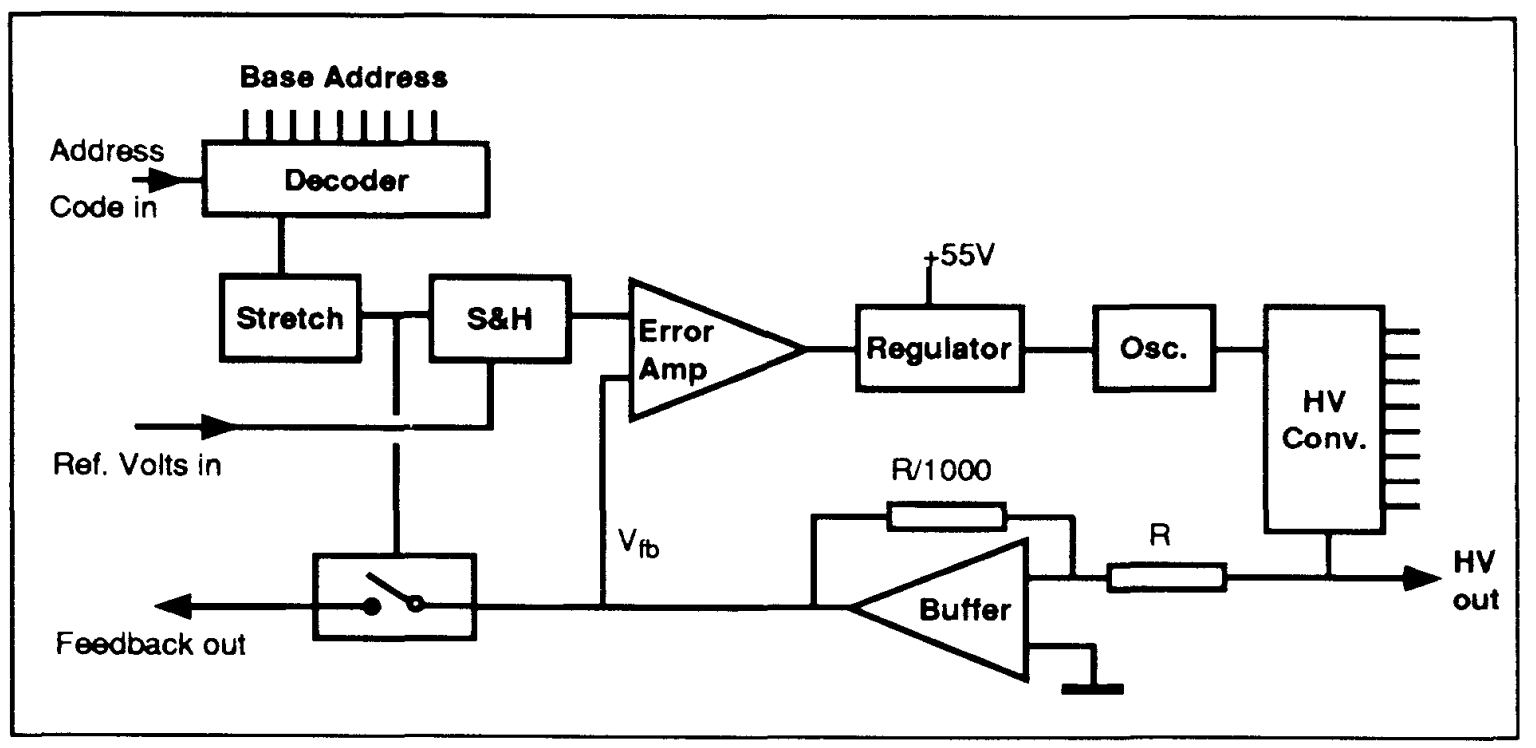

Figure 1: Working principle of the $\mathrm{HV}$ base

HV controller (HIVOC) is sampled and stored in a sample and hold amplifier on the receiver card. At the same time, the output of the buffer amplifier is connected to the feedback line and an analog signal of $-1 / 1000$ of the cathode voltage is sent back and measured by the HIVOC. This feedback voltage is used to check the performance of the base. The controller cycles through all bases of one group (up to 512 bases) with a refresh cycle of 1 second. The reference voltage $V_{\text {ref }}$ stored in the sample and hold and the feedback voltage $V_{\mathrm{fb}}$ generated by the buffer are compared by an error amplifier, whose output drives the regulator. The regulator circuit determines the amplitude of the square wave of the $200 \mathrm{kHz}$ oscillator (up to the supply voltage of $55 \mathrm{~V}$ ), which finally drives the Greinacher cascade.

The bases of one group are linked together by a common bus cable (flat cable) of 6 lines:

- $+5 \mathrm{~V}$ supply voltage for the on-board electronics

$-+55 \mathrm{~V}$ supply voltage for the oscillator

- address line (serial code)

- reference line (analog signal for the demand or reference voltage)

- feedback line (analog signal for control purposes)

- ground line

To avoid distortions of the serial code and the analog signals $\left(\mathrm{V}_{\mathrm{fb}}\right.$ and $\mathrm{V}_{\text {ref }}$ ), shielded twisted pair cables are used for the main distance of about $40 \mathrm{~m}$ from the control room to local repeater boxes. The two supply voltages $(+5 \mathrm{~V}$ and $+55 \mathrm{~V})$ are added at the repeater boxes, placed close to the detector.

Details on the technical features of the bases and results from test measurements with prototypes are given by L. Hubbeling [6]. 


\section{The Greinacher voltage multiplier}

The Greinacher type on-board voltage multiplier is a miniaturized version of the generator commonly used for Cockcroft-Walton accelerators [7].

The design of the Greinacher cascade is optimized for the russian type (FEU-84) "Venetian Blind" 1.3-inch PMs used in the experiment. As the operating voltages of these PMs range between $1400 \mathrm{~V}$ and $1900 \mathrm{~V}$ the PM base should be able to provide cathode voltages of about $2000 \mathrm{~V}$. The recommended voltage distribution for the PM (from cathode via grid and 12 dynodes to anode) is given by:

$2.75: 1.4: 1: 1: 1: 1: 1: 1: 1: 1: 2.1: 2.3: 2.75: 3$.

Instead of a resistive divider chain, the cathode, grid and dynode voltages are taken directly from the different stages of the Greinacher cascade and the recommended voltage distribution can be fairly well approximated by the following number of cascade stages $6+3+2+2+2+2+2+2+2+2+3+4+5+6=43$ in total. Therefore, the Greinacher cascades used for our PM bases is made of 43 stages. With a maximum regulator output of about $52 \mathrm{~V}$ the $\mathrm{HV}$ is limited to $43 \times 52$ $\mathrm{V}=2250 \mathrm{~V}$.

In total, about $11,000 \mathrm{HV}$ cards were produced by industry in surface mount technology (SMT) and the same number of receiver cards were equipped with standard components. It should be mentioned that the first $10,000 \mathrm{HV}$ cards show a lower HV output due to the bad quality, i.e. a significant drop of the capacitance with bias, of the chosen capacitors. The maximum HV is therefore limited to $1900 \mathrm{~V}$ (instead of $2250 \mathrm{~V}$ ), but still sufficient, since the average voltage applied to our PMs is about $1600 \mathrm{~V}$. However, all prototypes and several hundred spare bases, produced in addition with better capacitors, provide the expected $2250 \mathrm{~V}$ without any problems. Even if the gain of some PMs should drop in the future, the existing HV system is able to provide the required voltages with a reasonable safety margin.

Due to current consumption and the recovery current of the diodes on the HV card, the deviations of the dynode voltages from their design values increase with the number of cascade stages but remain for $\mathrm{HV}$ cards with high quality capacitors within a few per cent. The HV output for typical cathode voltages $(1600 \mathrm{~V})$ is stable up to currents at the 12 th dynode of about $1 \mathrm{~mA}$ (somewhat less for the series of 10,000 bases with poor capacitors). A simple current limiter on the base itself protects the PMs from overload as e.g. caused by hazardous light exposure.

Due to the dense packing of the detector in groups of $4 \times 6$ single modules, epoxied together in self contained subdetectors ("supermodules") with practically no dead space in vetween, severe geometrical constraints limited the dimensions of the PM bases. The overall dimensions of the PM bases which are rigidly connected with the sockets of the PM tubes are only $16 \times 3.5 \times 1.5 \mathrm{~cm}^{3}$. Especially, the width of 3.5 $\mathrm{cm}$ is limited by the dimensions of the single leadglass modules of $4 \times 4 \mathrm{~cm}^{2}$ cross section. The height of only $1.5 \mathrm{~cm}$ is achieved by mounting both PC-boards ( HV and receiver card) on top of each other with $3 \mathrm{~mm}$ spacing in between. 
HV stability:

The HV stability of the system can be tested easily by monitoring the feedback voltages of the bases. During long-term-stability tests of several days duration with operating voltages of $1900 \mathrm{~V}$, the deviations from the demand voltages were measured and a $<\sigma_{\mathrm{HV}}>=0.5 \mathrm{~V}$ was found, i.e: in average, the relative deviation is less than $3 \times 10^{-4}$.

Noise:

In early prototypes of the PM bases, it was found that the high frequency of the oscillator with fast rise and fall times and large amplitudes can cause crosstalk to the signal lines. Improvements of the layout, especially a complete separation of the signal line (standard lemo cables and connectors) from the base, improved ground to ground connections between both $\mathrm{PC}$-boards and special choice of some of the crucial components led to a significant reduction of this crosstalk. Although no additional shielding is used, except for the metallic cover of the supermodules, the $200 \mathrm{kHz}$ noise signal at $50 \mathrm{Ohm}$ is now less than $2 \mathrm{mV}$ (peak-to-peak), causing only a negligible broadening of pedestals and resolution degradation. The ripple of the $\mathrm{HV}$ was measured to be about $200 \mathrm{mV} \mathrm{[6].}$

Power consumption:

In contrast to conventional resistive divider chains, the current is directly taken from the different cascade stages, significantly reducing the power consumption and dissipation. The power consumption, measured during the calibration of the detector and corresponding to an average $\mathrm{HV}$ of about $1600 \mathrm{~V}$ is $10 \mathrm{~mW}$ per channel at the $5 \mathrm{~V}$ and $65 \mathrm{~mW}$ per channel at the $55 \mathrm{~V}$ line (losses in the power cables and the repeater boxes already included). The power dissipation in the detector is therefore less than $75 \mathrm{~mW}$ per channel, or 750 Watts for all 10,080 channels and thus requires no cooling.

\section{The VME-based HV controller}

The HV controllers are a set of control cards (HIVOCs) which fit into a double height VME crate. Each of these single slot VME cards is equipped with $4 \mathrm{HV}$ drivers, controlled by a single Motorola MC68332 microprocessor. In total, each HIVOC is able to drive and control $4 \times 512=2048 \mathrm{HV}$ bases.

Due to the segmentation of the leadglass calorimeter, it was convenient to define 20 supermodules i.e. 480 channels as one group. For the control of all 10,080 channels of our experiment, we therefore need only 6 HIVOCs, occupying less than half of a VME crate. A schematic view of the VME-based HV controller is shown in Fig.2. 


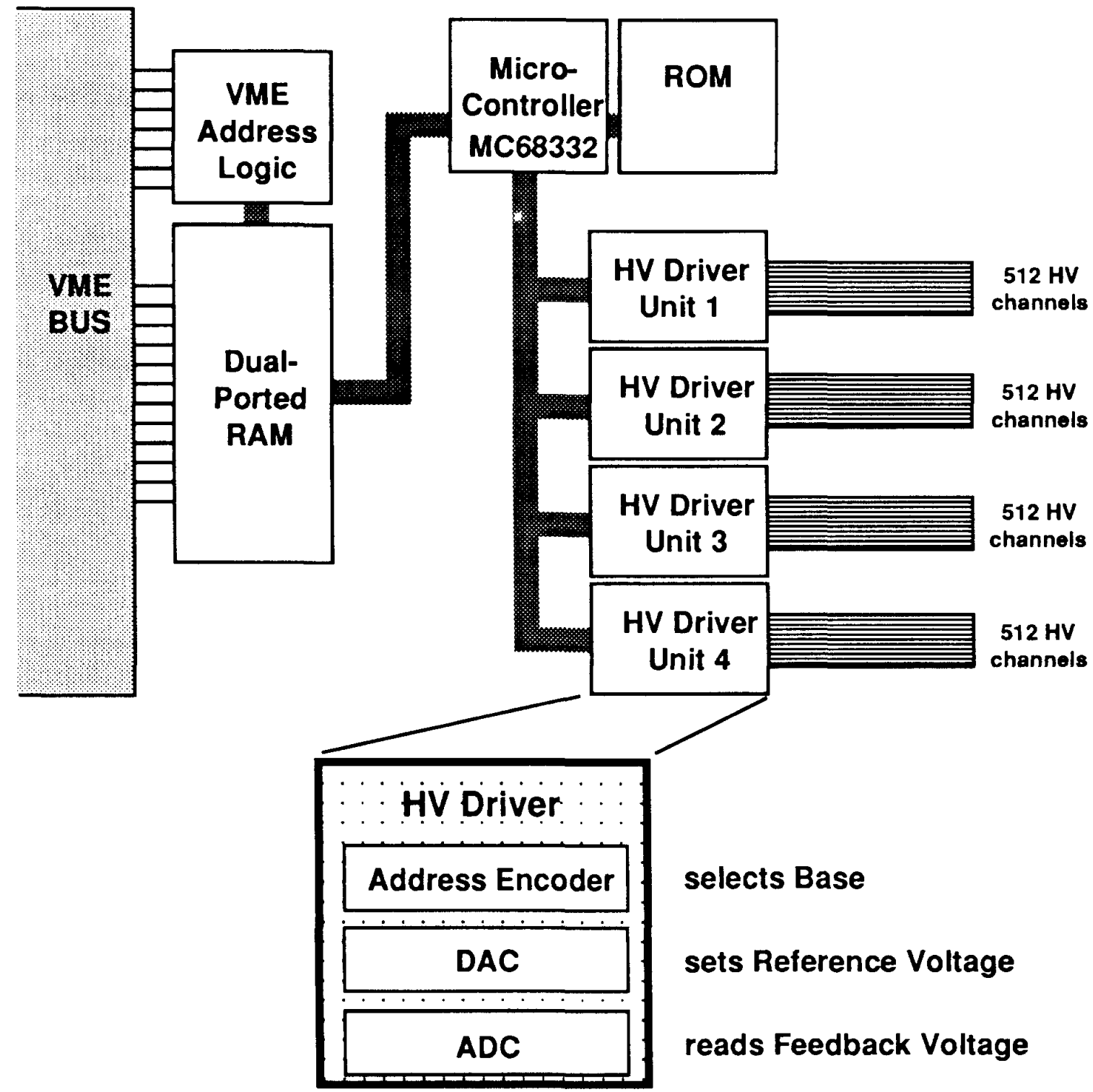

Figure 2: Block diagram of the VME-based HV controller 
Each of the 4 drivers of the HIVOC consists of a 12-bit DAC that sets the reference voltage, a 12-bit ADC that reads the feedback voltage, and a serial encoder that generates the code to address a particular base. Under the control of the microprocessor, the driver continuously cycles through all bases of a group and refreshes and verifies the voltage settings, once per second.

The user interface to the HIVOC is a section of $64 \mathrm{~K}$ dual-ported RAM that can be accessed from the VME bus and by the microprocessor. The memory map maintained by the HIVOC contains for each channel the demand or reference voltage $\left(V_{\text {ref }}\right)$, the feedback voltage $\left(V_{f b}\right)$, the last 10 differences of the demand and the feedback voltages as well as the average of these differences, a fault counter indicating the number of successive wrong feedback voltage readings, the list of bad channels and status and control registers. Usually, the demand voltages are written and the "list of bad channels" is periodically reviewed by a VME processor that runs a higher-level HV control program. The control and status register section of the dual-ported RAM allows to control and tune the overall behavior of the HIVOC controller and query the status of most of the components. Some of the options are, e.g.: switching on/off the HVs for each driver, to query the status of the processor and examine the reason for the most recent reset, to adjust the allowed deviations between $V_{\text {ref }}$ and $V_{f b}$, which define a bad channel and enable the controller to shut down bad channels for safety reasons. A detailed description of the VME high voltage control card is given by $T$. Hubbeling [8].

\section{Test measurements}

A series of test measurements has been performed during the development and the production phase of the bases $[9,10]$. Some of the results are already discussed in the previous sections and a comprehensive description of the basic features and the performance of single bases is given by L. Hubbeling [6]. A special measurement was performed to study the gain stability of the bases as a function of the length of the refresh cycle, essentially testing the accuracy and stability of the sample and hold circuit. As a result, up to cycle lengths of $1 \mathrm{sec}$, no periodic gain variations were found [11]. In this section we focus on test measurements performed with various leadglass detectors and electron beams from $2 \mathrm{GeV}$ to $20 \mathrm{GeV}$ at the CERN SPS. The electron beams are used to generate electromagnetic showers as produced by high-energy photons in the heavy-ion experiments. The electron-positron-pairs of the electromagnetic shower cause the emission of Cerenkov light in groups of adjacent leadglass modules, which in turn (guided by total reflection) is read out by the photomultiplier tubes. The longitudinal dimension of the leadglass modules corresponds to about 14 radiation lengths. The lateral dimensions of $4 \mathrm{~cm} \times 4$ $\mathrm{cm}$ are close to the Molière radius of the leadglass of $R_{m}=3.68 \mathrm{~cm}$. Position resolution is achieved by the "center-of-gravity" method and the total energy of the shower is calculated from the weighted sum of neighboring modules containing the electromagnetic shower. For a detailed discussion of the methods of particle identification in modular electromagnetic calorimeters see $[12,13]$. 
The energy resolution is commonly expressed in terms of $\sigma(\mathrm{E}) / \mathrm{E}=\mathrm{c} 1 / \sqrt{\mathrm{E}}+\mathrm{c} 2$. The energy resolution of the WA98 leadglass spectrometer, equipped with the new HV system, has been measured at the CERN SPS with electron beams of various energies between $2 \mathrm{GeV}$ and $20 \mathrm{GeV}$ (Tab. 1).

\begin{tabular}{|c|c|}
\hline electron energy & energy resolution [\%] \\
\hline \hline $2 \mathrm{GeV}$ & $5.48 \pm 0.40$ \\
$3 \mathrm{GeV}$ & $4.01 \pm 0.40$ \\
$5 \mathrm{GeV}$ & $3.27 \pm 0.10$ \\
$10 \mathrm{GeV}$ & $2.63 \pm 0.08$ \\
$20 \mathrm{GeV}$ & $2.00 \pm 0.13$ \\
\hline
\end{tabular}

Tab. 1. Energy resolution of the WA98 leadglass spectrometer, taken from [15].

In Tab. 2, a comparison between similar leadglass detectors is made, indicating the good quality of the new detector and the high stability of the new HV system. The energy resolution achieved with the WA98 leadglass detector is similar to the very good result obtained with a small test setup of $5 \times 5$ leadglass modules and resistive divider bases at the AGS in 1990 [14] and slightly better than the energy resolution of $2.8 \%$ at $10 \mathrm{GeV}$ achieved with the "Towers" (leadglass arrays with the same modules as used for WA98) of the WA80 experiment [16].

It should be mentioned, that the greater length of the leadglass modules of the Saphir detector (and hence a lower leakage of the electromagnetic shower) leads to a better energy resolution, especially for high energies [12]. A detailed description of the measurements of WA98 is given in [15].

\begin{tabular}{|l|c|c|c|c|}
\hline detector & leadglass & length & relative energy resolution & Ref. \\
\hline \hline Saphir & SF5 & $18 \mathrm{X}_{0}$ & $\sigma(\mathrm{E}) / \mathrm{E}=(6.0 \pm 1.0) \% / \sqrt{\mathrm{E}[\mathrm{GeV}]}+(0.4 \pm 1.0) \%$ & {$[12]$} \\
Test setup & TF1 & $14 \mathrm{X}_{0}$ & $\sigma(\mathrm{E}) / \mathrm{E}=5.0 \% / \sqrt{\mathrm{E}[\mathrm{GeV}]}+1.4 \%$ & {$[14]$} \\
WA98 & TF1 & $14 \mathrm{X}_{0}$ & $\sigma(\mathrm{E}) / \mathrm{E}=(5.5 \pm 0.6) \% / \sqrt{\mathrm{E}[\mathrm{GeV}]}+(0.8 \pm 0.2) \%$ & {$[15]$} \\
\hline
\end{tabular}

Tab. 2. Energy resolution for similar leadglass detector arrays.

Summarized, the energy resolution of the WA98 experiment using the new HV system is as good or even better than the one obtained with comparable leadglass arrays and conventional PM bases with resistive divider chains. Moreover, the calibration of the leadglass spectrometer in spring 1994 has shown that the full system with 10,080 bases can be run and monitored successfully with the new HV system. 


\section{Summary and Conclusions}

A new high voltage power supply for the 10,080 PMs of the leadglass calorimeter of the WA98 experiment at CERN has been built and successfully tested at the CERN SPS. The main advantages of the new system consisting of 10,080 active PM bases with on-board Greinacher voltage multipliers and a VME-based remote HV control, are considerable and can be summarized as follows:

- a very low heat dissipation of about $75 \mathrm{~mW}$ per channel (less than $10 \%$ of that of conventional systems) allowing the operation of densely packed calorimeters without cooling,

- a tremendously reduced amount of cabling with the complete elimination of HV cables,

- reduced costs per channel for the overall system,

- an easy way of setting and controlling the voltages

Compared to similar developments, the use of a common bus system and the new controllers, that address and cycle through all bases, significantly reduced the space required for the control units to less than half a VME crate for 10,000 channels.

The energy resolution of the leadglass spectrometer with the new HV power supply was found to be slightly improved compared to that obtained with conventional HV systems using resistive divider chains.

The new concept, suggested and developed by L. Hubbeling $[1,6]$, is well suited for large PM systems. For future detector developments, similar high voltage systems should be envisaged, especially, when even larger arrays and low power dissipation are required, as e.g. for the various detectors of the $\mathrm{LHC}$. 


\section{Acknowledgements}

We would like to sincerely thank L. Hubbeling who provided us with the design of the HV bases and for the many valuable discussions and his support during the development and the production of the new high voltage system, and C. Urra, $\mathrm{N}$. Heine, A. Przybyla and W. Verhoeven for their considerable contributions to the successful assembly of the leadglass detector. We further like to thank Prof. Klanner who encouraged us to this project.

\section{References}

[1] L. Hubbeling, A low power, high frequency DC-DC converter for generating high voltages, CERN/EP Internal Report 78-5, 1978.

[2] B. Lu, L.W. Mo and T.A. Nunamaker, The Cockcroft-Walton Photomultiplier Tube Base and the Ethernet High Voltage Controller, Report VPI-IHEP-91/1.

[3] S. Ahn et al., A new VME based High Voltage Power Supply for Large Experiments, FERMILAB-Conf-91/310.

[4] Zeus Status Report 1993.

[5] Experiments at CERN in 1993/1994, Proposal for a large acceptance hadron and photon spectrometer, CERN/SPSLC 91-17.

[6] L. Hubbeling, Large Photomultiplier Systems - A New Approach, CERN/ECP 92-10

[7] J.D. Cockcroft and E.T.S. Walton, Proc. Roy. Soc. (London) A137, 229(1932).

[8] T. Hubbeling and L. Hubbeling, HIVOC, a VME High-Voltage Control Card for large Photomultiplier Systems, CERN/ECP 93-2.

[9] GSI Scientific Report 1991; A Photomultiplier Supply for Large Systems (p. 383).

[10] GSI Scientific Report 1992; Development and Test Measurements of a New HV Supply for Large Photomultiplier Systems GSI (p. 397).

[11] M.L. Purschke et al., A new high voltage supply for large photomultiplier systems, Proceedings of the Third International Conference on Electronics for Future Colliders, Ed. by G. J. Blanar and R. L. Sumner, Chestnut Ridge, NY, 1993. p. 237-246.

[12] H. Baumeisier et al., Nucl. Instr. and Meth. A292 (1990) 81.

[13] F. Berger et al., Particle identification in modular electromagnetic calorimeters, Nucl. Instr. and Meth. A321 (1992) 152.

[14] V. Manko et al., Test performed on the Russian Pbgl, Phenix Technical Notes, Vol. 3, PN12 (1992).

[15] G. Schepers, Entwicklung und Erprobung eines LED-gestützten Monitorsystems für ein modulares Bleiglasspektrometer, Diplomarbeit, Universität Münster, 1994.

[16] T. Peitzmann, private communication (1994). 